\title{
Generation of acoustic helical wavefronts using metasurfaces
}

\author{
Hussein Esfahlani* and Herve Lissek ${ }^{\dagger}$ \\ Signal Processing Laboratory (LTS2), Ecole Polytechnique Fédérale de Lausanne (EPFL), CH-1015 Lausanne, Switzerland
}

Juan R. Mosig

Laboratory of Electromagnetics and Antennas (LEMA), Ecole Polytechnique Fédérale de Lausanne (EPFL), CH-1015 Lausanne, Switzerland

(Received 12 July 2016; revised manuscript received 17 November 2016; published 30 January 2017)

\begin{abstract}
It has been shown that acoustic waves with helical wavefronts can carry angular momentum, which can be transmitted towards a propagating medium. Such a wave field can be achieved by using a planar array of electroacoustic transducers, forming a given spatial distribution of phased sound sources which produce the desired helical wavefronts. Here, we introduce a technique to generate acoustic vortices, based on the passive acoustic metasurface concept. The proposed metasurface is composed of space-coiled cylindrical unit cells transmitting sound pressure with a controllable phase shift, which are arranged in a discretized circular configuration, and thus passively transforming an incident plane wavefront into the desired helical wavefront. This method presents the advantage of overcoming the restrictions on using many acoustic sources, and it is implemented with a transmitting metasurface which can be easily three-dimensionally printed. The proposed straightforward design principle can be adopted for easy production of acoustic angular momentum with minimum complexity and using a single source.
\end{abstract}

DOI: 10.1103/PhysRevB.95.024312

The discovery that waves can carry angular momentum may date back to Poynting and his claim on angular momentum of the circularly polarized light [1], which was later validated experimentally [2]. While the angular momentum due to the circular polarization is linked to the photon spin, there is another type of angular momentum due to the spatial distribution of electromagnetic waves which is called orbital angular momentum (OAM) [3]. In 1992, Allen et al. studied the mechanical torque resulting from electromagnetic orbital angular momentum waves [4], which has led to extensive theoretical and experimental studies on optical trapping, tweezers, spanners, etc. [5].

Although acoustic waves are not naturally polarized to produce spin angular momentum, they still can carry orbital angular momentum. The wave field singularity or a dislocation in the wavefront as a key feature of wave vortices was first studied in 1979 for acoustic waves [6]. Twenty years later, Hefner et al. produced the first ultrasonic beam with helical phase and screw dislocation [7]. Moreover, it was shown using the concept of pseudomomentum that acoustical vortices have the same properties as their optical counterparts. Thus, for a pressure wavefront with a phase dependence of $e^{j l \phi}$ (with $\phi$ and $l$ being the azimuthal angle and the topological charge, respectively), the torque is proportional to the absorbed power and to the $l / \omega$ ratio, where $\omega$ is the angular frequency [8]. Thereafter, different studies were conducted on the theoretical and practical aspects of acoustic angular momentum and screw-type

\footnotetext{
*Corresponding author: hussein.esfahlani@epfl.ch

${ }^{\dagger}$ Corresponding author: herve.lissek@epfl.ch
}

Published by the American Physical Society under the terms of the Creative Commons Attribution 4.0 International license. Further distribution of this work must maintain attribution to the author(s) and the published article's title, journal citation, and DOI. dislocations [9-14]. Moreover, the transfer of acoustic angular momentum to matter has been empirically validated [15-20].

Several methods have been proposed to create acoustic helicoidal waves, carrying angular momentum. Hefner et al. used an active polyvinylidene fluoride (PVDF) with a tangential helical surface supported by a twisted ring to create the first sonic helicoidal beam [7]. Later, Ealo et al. improved this configuration using ferroelectrets [21]. However, a vertical dislocation of $1 \lambda$ is required in the twisted ring (transducer diaphragm) and this technique is only suitable for ultrasounds. Hefner et al. proposed then an alternative technique, based on a circular array of phase-shifted transducers [22]. This method has been adopted since then in most of the studies to create acoustical vortices, requiring many transducers which are individually controlled [23]. Gspan et al. used optoacoustics for the generation of ultrasonic screw dislocations [24]. They used a pulsed laser beam to illuminate an absorbing spiral phase plate which ultimately produces ultrasonic pulses with a helical phase. Besides being complicated, this is a low-efficiency procedure which needs high-power short laser pulses. Naify et al. used leaky-wave antenna to create helical waves; although this method leads to very compact structures, it can create a nonuniform wavefront due to the difference in the amount of leaked power between input and output [25]. Jiang et al. [26] and Ye et al. [27] used the acoustic Helmholtz resonators to get acoustic OAM and were able to decrease the thickness of OAM metasurfaces down to $\lambda / 2$. Recently, Wang et al. proposed the use of a spiral shape structure to get acoustic vortices in ultrasonic frequencies [28].

A close study of the aforementioned techniques reveals that to generate acoustic vortices, the wavefront of the acoustic waves should be engineered to produce the phase dependence of the form $e^{j l \phi}$. Here, we introduce a method to create acoustic helical waves based on acoustic metasurfaces with acoustic space-coiled unit cells, which decreases the thickness of acoustic OAM generators down to $\lambda / 3$. Metasurfaces are artificially engineered surfaces composed of unit cells which 
manipulate the reflected and/or transmitted wavefronts in a preferred, predefined, and unconventional way $[29,30]$. We begin by introducing a unit-cell design based on the space-coiling technique [31-35]. This labyrinthine-type unit cell allows an efficient use of space [36]. Moreover, it can be analytically defined and this facilitates the design and optimization procedure. The proposed unit cell is used for the phase coding of a digitized surface, which converts acoustic plane wavefronts to acoustic helical wavefronts. Finally, the structure has been fabricated through three-dimensional (3D) printing and experimental results have validated the theoretical proposal.

Unit cell. To achieve a labyrinthine unit cell, a helicoid geometry is defined so as to use space most efficiently. The helicoid is a ruled surface that is described by a straight line rotating at a given angular rate around a fixed axis [37]. Let us suppose we have a cylindrical tube which intersects a helicoid. The resulting geometry is a space-coiled structure which connects the two ends of the cylindrical tube through two helical acoustic paths, longer than the physical length of the tube. Moreover, each transversal cross section of the proposed geometry is composed of two fluid-filled half disks, separated by the helicoid. This means that unlike the previous spatially modulated space-coiled structures reported so far, the whole cross section of the tube is used to transmit sound waves through the structure. These unit cells allow the design of a phase-coded metasurface with transmission phase profile of $(0-2 \pi)$ and near unitary amplitude. It is obvious that the phase of the transmitted wave depends on the effective length of the twisted ducts. Thus, the transmission phase can be engineered by modifying the number of helicoid turns along the tube. However, the presence of the helicoidal wall at the entrance of the duct results in a discontinuity between the outer and inner medium, which leads to an impedance mismatch. Moreover, such impedance mismatch may be further increased by the densification of the labyrinthine path and an increase in the number of turns. To mitigate this issue, the twisting profile of the helicoid is modulated along the length of the structure using piecewise functions, allowing optimization of the transmission coefficient in terms of phase and amplitude.

The helicoid can be mathematically described as the following parametric equation:

$$
\begin{aligned}
\vec{r}(\rho, t)= & \langle x, y, z\rangle, \\
= & \left\langle\rho \cos \left[2 \pi \int_{0}^{t} f(\tau) d \tau\right],\right. \\
& \left.\rho \sin \left[2 \pi \int_{0}^{t} f(\tau) d \tau\right], b t\right\rangle,
\end{aligned}
$$

where $\rho$ is the radius of helicoid, $b$ is the constant rate of gradual displacement along the $z$ axis, and, for $b=1, t$ defines the height of the unit cell. The spatial modulation function of the helicoid is $f(\tau)=f_{c}+f_{\Delta} x_{m}(t)$, where $f_{c}$ is the average spatial frequency of twists, $f_{\Delta}$ is the deviation from $f_{c}$, and $x_{m}(t)$ is a piecewise function allowing changing the spatial variations. Therefore, $f_{c}$ controls the phase of the transmission coefficient, whereas $f_{\Delta}$ and $x_{m}$ have an influence on the amplitude of the transmission coefficient, acting on

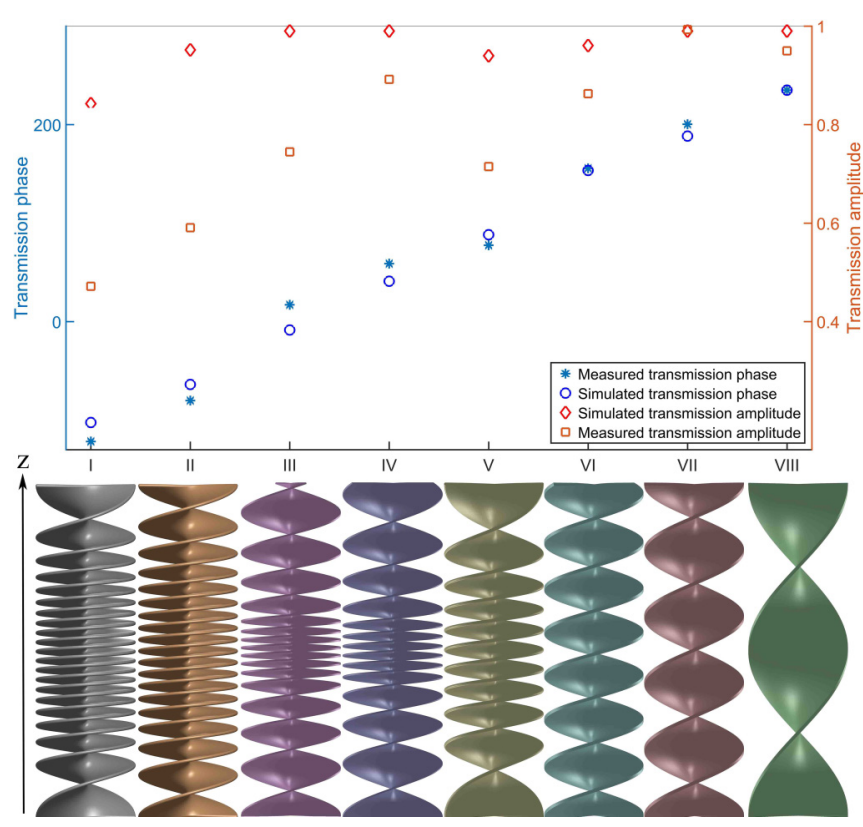

FIG. 1. Top: Sound-transmission coefficient phase (blue) (in degrees) and amplitude (red). Bottom: Geometries of the eight simulated configurations [densely twisted helicoids (left) and coarsely twisted helicoids (right)].

the impedance matching (see Table 1 in the Supplemental Material [38]).

Figure 1 (bottom) represents eight spatially modulated helicoids. The geometrical modulations of these twisted surfaces, hosted inside cylindrical tubes, are described by piecewise functions $f(\tau)$ (defined in the Supplemental Material [38]), which have been optimized to provide a smooth transition between the sound pressure at the input $\left(p_{i}\right)$ and at the output $\left(p_{t}\right)$, focusing on improving the impedance matching and on ensuring the near unity transmission coefficient as required for such metasurface unit cells. These unit-cell prototypes have been 3D printed using selective laser sintering (SLS), and the sound-transmission coefficient $p_{t} / p_{i}$ of each unit cell has been measured in a standing-wave tube (see Figs. 1 and 2 in the Supplemental Material [38]). The resulting sound-transmission coefficient amplitudes and phases are reported in Fig. 1 (top) and compared to simulations of the same structures obtained with COMSOL MULTIPHYSICS for the operating frequency of $1060 \mathrm{~Hz}$.

As can be seen in Fig. 1 (top), the measured soundtransmission coefficient phases of the fabricated prototypes agree well with the numerical results for all unit cells, spanning the $(0-2 \pi)$ range with $\pi / 4$ phase steps, whereas the transmission presents almost near-unity amplitudes only for the coarser unit cells (IV to VIII). For unit cells I-III, the measured amplitudes decrease as the number of helicoidal turns increases, and discrepancies between simulation and measurement results increase. The observed differences, which are affecting the denser unit cells, are due to several design simplification that are listed in the following. In the numerical simulation, the helicoidal and cylindrical walls have been considered smooth surfaces with zero thickness presenting hard boundary conditions, and the thermoviscous losses have 


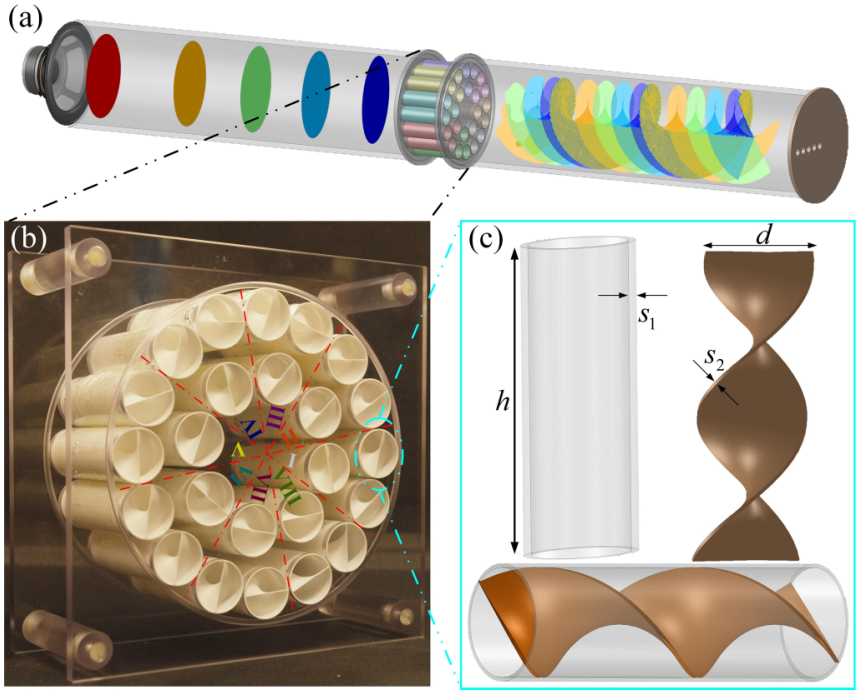

FIG. 2. (a) Measurement setup for the assessment of the metasurface, with an illustration of the resulting acoustic wave structure in the upstream and the downstream. (b) 3D-printed metasurface prototype composed of eight circular sectors (dashed red lines) decorated with three unit cells in each sector. Numbers I-VIII correspond to helicoidal unit cells designed in Fig. 1. (c) Helicoidal unit cells with global dimensions: $d=30 \mathrm{~mm}, h=100 \mathrm{~mm}, S_{1}=1 \mathrm{~mm}, S_{2}=0.5 \mathrm{~mm}$, and different levels of space coiling.

been neglected, for the sake of reducing the computational cost. However, in the actual 3D-printed prototype, the helicoidal and cylindrical walls are nonsmooth and porous, presenting an irregular surface state with an average thickness of 0.5 and $1 \mathrm{~mm}$, respectively (considering the fabrication tolerances, this value is unpredictable and can be thinner or thicker than this average), and are likely to vibrate, thus reducing the transmitted sound energy. Moreover, in 3D-printed prototypes using SLS, the remaining building powder that inevitably remains in the labyrinthine paths after construction can be cleaned for coarse unit cells using compressed air, whereas it is difficult, if not impossible, for denser twisted shapes. Consequently, the remaining powder can affect the soundtransmission performance of the denser unit cells. Having stated the physical source of deviation between the simulation and measurements, the following phenomena can explain how they affect the sound-transmission amplitude:

(i) The numerical simulations have neglected, in a view to reducing computational cost, the thickness of helicoidal walls, as well as the obstruction to sound propagation due to the remaining powder in the denser unit cells which is not possible to account for. These two phenomena affect the transmission performance and result in a significant amount of the sound intensity reflected back toward the input of the channel. Intuitively, these sound intensity reflections are likely to be dominant in the denser unit cells and almost vanish for coarser labyrinthine paths (this can be quantitatively verified in Fig. 3 in the Supplemental Material [38]).

(2) The numerical simulations have also neglected the thermoviscous losses due to the rough, porous surfaces of the labyrinthine structure, as well as dissipation associated with the remaining excess powder of the fabrication process, which
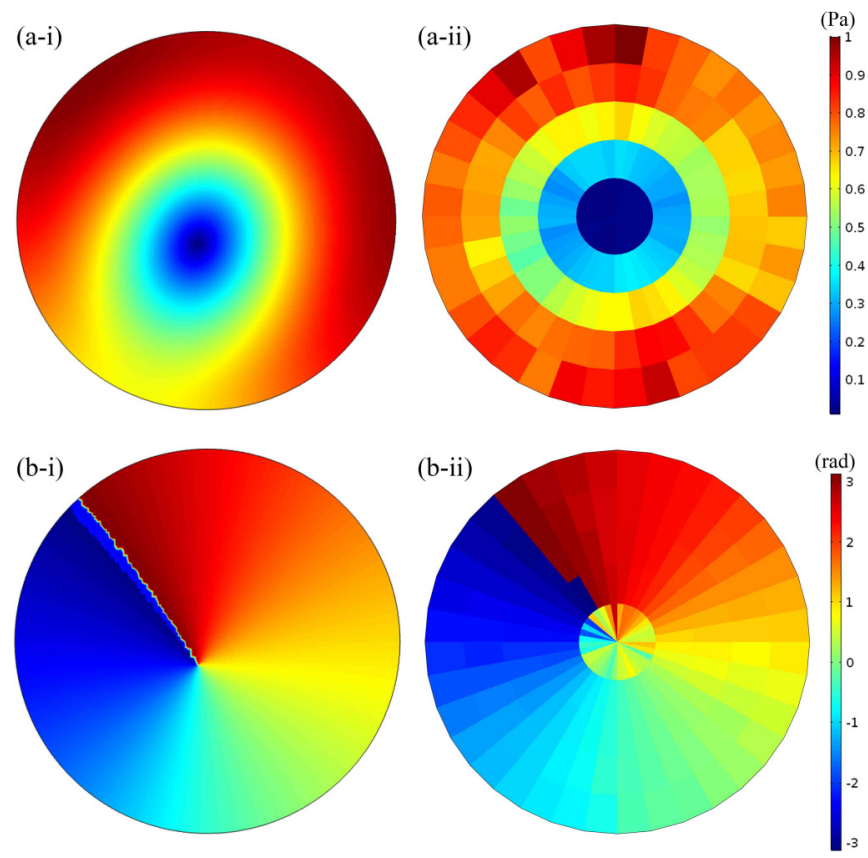

FIG. 3. Simulated and measured acoustic field distribution in the transversal plane, at frequency $f=1060 \mathrm{~Hz}$. (a) Amplitude and (b) phase for (i) simulation and (ii) measurement.

increases for denser unit cells due to the longer and narrower paths.

(3) Last, the thin walls of the unit cells are likely to vibrate that may explain the overestimation of the transmitted power in the simulation.

Higher transmission coefficient amplitude could be achieved by increasing the length of the unit-cell tube $h$, which increases the overall metasurface thickness. Moreover, the definition of different piecewise functions $f(\tau)$ for the modulation of helicoids could be improved to optimize the performance of the denser unit cells in terms of transmission coefficient amplitude.

OAM metasurface. To convert a plane wavefront from one upstream propagating medium into a helicoidal wavefront carrying orbital angular momentum in a downstream medium, a specific interface with a surface-dependent transmission phase should be designed. For that purpose, a metasurface with a circular arrangement of unit cells is proposed, the transmission phase of which is set according to the design presented in the preceding section. To achieve the acoustic vortices with a topological charge of integer value $l$, the proposed circular configuration should cover the phase range of $(0-2 \pi l)$, that is, the unit cells in the transmission phase range $(0-2 \pi)$ should intermittently cover the circular arrangement of the metasurface for $l$ times. Let us consider a circular surface which is segmented into $M$ equal sectors, with each sector being composed of $m$ equal and equidistant unit cells with circular cross sections. Then, each of the $(M \times m)$ sections is individually phase coded using the designed labyrinthine-type unit cells. In the proposed configuration, $M$ controls the resolution of the generated helical wave, as well as its topological charge, and $m$ controls the amount of power which passes through each sector. For a metasurface with a given area, the amount of 
transmitted power can be balanced and made uniform by adjusting the density of unit cells per sector (number per sector). This can be used to correct the deviation of the metasurface output amplitude from the simulated OAM beam, which is due to the nonuniform transmission coefficients achieved on the unit-cell prototypes (see preceding section and Fig. 1).

Figure 2 depicts the acoustic OAM metasurface designed with helicoidal unit cells to convert plane wavefronts into acoustic helical waves with the topological charge of $l=1$. The surface is divided into $M=8$ circular sectors, each representing transmit coefficients with the phase shift of $\pi / 4$ with respect to its adjacent sectors, and each sector is filled by $m=3$ similar unit cells as designed in Fig. 1 . While the presented example in this study targets $l=1$, the metasurface can be designed to achieve higher values of the topological charge $l$ provided that the proposed unit cells in $M$ sectors encompass the phase $(0-2 \pi l)$ for every circular row.

Figure 2(a) illustrates the experimental setup composed of two waveguides separated by the proposed metasurface prototype. The metasurface consists of an array of $M \times m$ regularly spaced labyrinthine unit cells $(h=100 \mathrm{~mm})$, squeezed by two square plates. The plates have dimensions $240 \times 240 \times 8$ $\mathrm{mm}$ and are made out of acrylic glass, with circular holes of diameters $d=30 \mathrm{~mm}$. The holes are drilled equidistantly in two concentric circular rows containing 8 and 16 holes, as depicted in Fig. 2(b). Each of the eight sectors of the metasurface is filled with three identical samples of one of the eight different unit cells shown in Fig. 2(c) and designed in the previous section. The metasurface is placed between two cylindrical tubes of inner diameter $194 \mathrm{~mm}$ and length $800 \mathrm{~mm}$. An electrodynamic loudspeaker is located at the termination of one duct (upstream), and the other termination is closed by an acrylic glass plate, holding a row of five PCB Piezotronics 130D20 1/4-inch measurement microphones. The microphone support is free to rotate around the waveguide axis in order to scan the whole cross section, thanks to a circular groove adapted to the periphery of the duct (see Table 2 and Figs. 4 and 5 in the Supplemental Material [38]). Finally, the perimeter of the tube is graded, and the acoustic wavefront is measured by spinning the measuring plate from $0^{\circ}$ to $360^{\circ}$. The measured sound pressure is then processed with a Bruel \& Kjaer Type 3160 Pulse multichannel analyzer. Although the closed termination presented by the microphone plate to the acoustic field yields standing waves, the acoustical vortices are still preserved and can be measured over the transversal plane.

The numerical assessment of the proposed prototype is done with commercial finite-element analysis software COMSOL MULTIPHYSICS, following a geometry similar to the one presented in Fig. 2(a), with the substitution of an anechoic condition for the microphone holder. All boundaries in the simulation setup are set to a "sound hard boundary condition," except the input and output of the test tube which are defined as pressure sound source and absorbing boundary condition, respectively. Finally, the "pressure acoustic" module is used in the frequency domain to simulate the structure.

Figure 3 illustrates the sound-pressure amplitude [Fig. 3(a)] and phase [Fig. 3(b)] over the microphone holder surface, both with measurements [Fig. 3(i)] and numerical simulation [Fig. 3(ii)]. The phase of the sound pressure is covering the full phase of $(0-2 \pi)$ over the cross section as expected for acoustic vortices, despite a singularity point (where all of the pressure phase is concentrated) slightly off center. This may be explained by the lack of uniformity of the transmission amplitudes observed within the different unit cells depicted in Fig. 1. In Fig. 3(b), the singularity point can also be visualized on the pressure amplitude distribution over the measured surface as the region where sound-pressure amplitude drops to zero. A close study of the measured pressure amplitude distribution reveals that opposite to the simulated results, the pressure dip is symmetrical and centered. This is due to the coarse spatial resolution of the measurement setup. Moreover, a phase ambiguity arises near the center [6]. The point with the lowest pressure amplitude also coincides with the point of phase singularity, in agreement with the theoretical descriptions of acoustic vortices. In these acoustic vortices, the amplitude distribution is expected to be symmetrically doughnut shaped. However, the simulated and measured data disagree in particular for the points further away from the center, where lobes of higher amplitude do appear. This is also explained by the nonuniform amplitudes of the unit cells and the discrepancies between the theoretically designed and practically implemented unit cells. The spatially shifted singularity point, as well as the asymmetry of the amplitude distribution in the wavefront, can be corrected by better aligning the transmission amplitudes.

Discussion. In this paper, we introduced a method to produce acoustic vortices based on acoustic metasurfaces which converts acoustic plane waves (generated from a single sound source) to helical waves. The proposed strategy relies on phase coding a surface using labyrinthine unit cells with a view to engineering the transmission phase through each unit cell. Moreover, the presented mathematical framework enables fast design and optimization and facilitates geometrical rendering, meshing, and numerical simulation. Spatial frequency modulation was used to improve the impedance matching. Additionally, the physical length of the proposed unit cell has been reduced to less than $\lambda / 3$, which is especially important for low-frequency sounds. Finally, 3D prototyping technology has been employed for easy fabrication of such a complex structure. The fast prototyping technique makes the proposed method a simple yet practical and economical way to generate helical waves which can be used to demonstrate sound-wave rotation. The easy production of acoustic vortices should open new horizons in many areas, such as transfer of acoustic angular momentum to matter, acoustic trapping, tweezers, spanners, and underwater alignment applications.

Acknowledgments. This research was supported by the Swiss National Science Foundation (SNSF) under Grant Agreement No. 200020-138086/1. The authors would like to thank Roland Dupuis, Pierre-André Pognant, and Karim Collomb of the AEM workshop for their help in the construction of the prototype.

H.E. proposed the idea and performed the design, numerical simulations, and experimental verifications. H.E., H.L., and J.M. prepared the manuscript and discussed the data. H.L. supervised the work closely and J.M. supervised the group. All authors contributed to review the manuscript. 
[1] J. H. Poynting, The wave motion of a revolving shaft, and a suggestion as to the angular momentum in a beam of circularly polarised light, Proc. R. Soc. London. Ser. A, Containing Papers of a Mathematical and Physical Character 82, 557, 560 (1909).

[2] A. Beth Richard, Mechanical detection and measurement of the angular momentum of light, Phys. Rev. 50, 115 (1936).

[3] J. D. Jackson, Classical Electrodynamics (Wiley, New York, 1999).

[4] L. Allen, M. W. Beijersbergen, R. J. C. Spreeuw, and J. P. Woerdman, Orbital angular momentum of light and the transformation of Laguerre-Gaussian laser modes, Phys. Rev. A 45, 8185 (1992).

[5] M. Padgett and R. Bowman, Tweezers with a twist, Nat. Photon. 5, 343 (2011).

[6] M. V. Berry, J. F. Nye, and F. J. Wright, The elliptic umbilic diffraction catastrophe, Philos. Trans. R. Soc. London A 291, 1382 (1979).

[7] B. T. Hefner and P. L. Marston, Acoustical helicoidal waves and Laguerre-Gaussian beams: Applications to scattering and to angular momentum transport, J. Acoust. Soc. Am. 103, 5 (1998).

[8] J.-L. Thomas and R. Marchiano, Pseudo Angular Momentum and Topological Charge Conservation for Nonlinear Acoustical Vortices, Phys. Rev. Lett. 91, 244302 (2003).

[9] R. Marchiano and J.-L. Thomas, Synthesis and analysis of linear and nonlinear acoustical vortices, Phys. Rev. E 71, 066616 (2005).

[10] J. Lekner, Acoustic beams with angular momentum, J. Acoust. Soc. Am. 120, 3475 (2006).

[11] J. Lekner, Angular momentum of sound pulses, J. Phys.: Condens. Matter 18, 6149 (2006).

[12] K. D. Skeldon, C. Wilson, M. Edgar, and M. J. Padgett, An acoustic spanner and its associated rotational Doppler shift, New J. Phys. 10, 013018 (2008).

[13] C. Wilson and M. J. Padgett, A polyphonic acoustic vortex and its complementary chords, New J. Phys. 12, 023018 (2010).

[14] L. Zhang and L. M. Philip, Angular momentum flux of nonparaxial acoustic vortex beams and torques on axisymmetric objects, Phys. Rev. E 84, 065601 (2011).

[15] A. O. Santillán and K. Volke-Sepúlveda, A demonstration of rotating sound waves in free space and the transfer of their angular momentum to matter, Am. J. Phys. 77, 209 (2009).

[16] K. Volke-Sepúlveda, A. O. Santillán, and R. R. Boullosa, Transfer of Angular Momentum to Matter from Acoustical Vortices in Free Space, Phys. Rev. Lett. 100, 024302 (2008).

[17] A. Anhäuser, Régis Wunenburger, and E. Brasselet, Acoustic Rotational Manipulation using Orbital Angular Momentum Transfer, Phys. Rev. Lett. 109, 034301 (2012).

[18] C. E. Demore, Z. Yang, A. Volovick, S. Cochran, M. P. MacDonald, and G. C. Spalding, Mechanical Evidence of The Orbital Angular Momentum to Energy Ratio of Vortex Beams, Phys. Rev. Lett. 108, 194301 (2012).

[19] Z. Hong, J. Zhang, and B. W. Drinkwater, Observation of Orbital Angular Momentum Transfer from Bessel-Shaped Acoustic Vortices to Diphasic Liquid-Microparticle Mixtures, Phys. Rev. Lett. 114, 214301 (2015).

[20] Régis Wunenburger, J. Israel, V. Lozano, and E. Brasselet, Acoustic orbital angular momentum transfer to matter by chiral scattering, New J. Phys. 17, 103022 (2015).

[21] J. L. Ealo, José Carlos Prieto, and F. Seco, Airborne ultrasonic vortex generation using flexible ferroelectrets, ultrasonics, ferroelectrics, and frequency control, IEEE Trans. 58, 1651 (2011).

[22] B. T. Hefner and P. L. Marston, An acoustical helicoidal wave transducer with applications for the alignment of ultrasonic and underwater systems, J. Acoust. Soc. Am. 106, 3313 (1999).

[23] L. Yang, Q. Ma, J. Tu, and D. Zhang, Phase-coded approach for controllable generation of acoustical vortices, J. Appl. Phys. 113, 4904 (2013).

[24] S. Gspan, A. Meyer, S. Bernet, and M. Ritsch-Marte, Optoacoustic generation of a helicoidal ultrasonic beam, J. Acoust. Soc. Am. 115, 1142 (2004).

[25] C. J. Naify, C. A. Rohde, T. P. Martin, M. Nicholas, M. D. Guild, and G. J. Orris, Generation of topologically diverse acoustic vortex beams using a compact metamaterial aperture, Appl. Phys. Lett. 108, 223503 (2016).

[26] X. Jiang, Y. Li, B. Liang, J.-c. Cheng, and L. Zhang, Convert Acoustic Resonances to Orbital Angular Momentum, Phys. Rev. Lett. 117, 034301 (2016).

[27] Y. Liping, Chunyin Qiu, Jiuyang Lu, Kun Tang, Han Jia, Manzhu Ke, Shasha Peng, and Z. Liu, Making sound vortices by metasurfaces, AIP Adv. 6, 085007 (2016).

[28] W. Tian, Manzhu Ke, Weiping Li, Qian Yang, Chunyin Qiu, and Z. Liu, Particle manipulation with acoustic vortex beam induced by a brass plate with spiral shape structure, Appl. Phys. Lett. 109, 123506 (2016).

[29] H. Esfahlani, S. Karkar, H. Lissek, and J. R. Mosig, Acoustic carpet cloak based on an ultrathin metasurface, Phys. Rev. B 94 014302 (2016).

[30] Y. X. Yangbo, W. Wang, H. Chen, A. Konneker, B.-I. Popa, and S. A. Cummer, Wavefront modulation and subwavelength diffractive acoustics with an acoustic metasurface, Nat. Commun. 5, 5553 (2014).

[31] L. Zixian and J. Li, Extreme Acoustic Metamaterial by Coiling Up Space, Phys. Rev. Lett. 108, 114301 (2012).

[32] Y. Li, B. Liang, Z.-M. Gu, X.-Y. Zou, and J.-C. Cheng, Reflected wavefront manipulation based on ultrathin planar acoustic metasurfaces, Sci. Rep. 3, 2546 (2013).

[33] K. Tang, C. Qiu, M. Ke, J. Lu, Y. Ye, and Z. Liu, Anomalous refraction of airborne sound through ultrathin metasurfaces, Sci. Rep. 4, 6517 (2014).

[34] Y. Li, X. Jiang, Rui-qi Li, B. Liang, Xin-ye Zou, Lei-lei Yin, and Jian-chun Cheng, Experimental Realization of Full Control of Reflected Waves with Subwavelength Acoustic Metasurfaces, Phys. Rev. Appl. 2, 064002 (2014).

[35] B. Yuan, Y. Cheng, and X. Liu, Conversion of sound radiation pattern via gradient acoustic metasurface with space-coiling structure, Appl. Phys. Exp. 8, 027301 (2015).

[36] E. Hussein, S. Karkar, and H. Lissek, On the design of unit-cells for acoustic metasurfaces, in Proceedings of the Ninth International Congress on Advanced Electromagnetic Materials in Microwaves and Optics-Metamaterials, Oxford, 2015 (IEEE, Piscataway, NJ, 2015), pp. 397-399.

[37] http://facstaff.susqu.edu/brakke/evolver/examples/periodic/ periodic.html; http://mathworld.wolfram.com/MinimalSurface. html (unpublished).

[38] See Supplemental Material at http://link.aps.org/supplemental/ 10.1103/PhysRevB.95.024312 for a detailed explanation of the $S$ parameters' retrieval procedure and the corresponding experimental method for assessing the transmission and reflection coefficients with four microphones, as well as the complete mathematical definitions of the helicoidal unit cells. 